\title{
Adaptation of Renal Microdialysis for Chronic Interstitial Collection of Angiotensin II and ATP
}

\author{
Priyanka Prathipati ${ }^{1}$, Syed Quadri ${ }^{1}$, Debra W. Jackson ${ }^{2} \&$ Keith E. Jackson ${ }^{1}$ \\ ${ }^{1}$ College of Pharmacy, University of Louisiana at Monroe, Monroe, LA, USA \\ ${ }^{2}$ College of Arts and Sciences, University of Louisiana at Monroe, Monroe, LA, USA \\ Correspondence: Keith E. Jackson, College of Pharmacy, University of Louisiana at Monroe, Monroe, LA \\ 71209-0497, USA. Tel: 1-318-342-1390. E-mail: kjackson@ulm.edu
}

Received: February 8, 2012 Accepted: February 25, 2012 Online Published: September 27, 2012

doi:10.5539/ijb.v4n4p127

URL: http://dx.doi.org/10.5539/ijb.v4n4p127

This research is supported by Board of regions (Research Competitiveness 2010)

\begin{abstract}
Numerous methods have been developed for in vivo collection, separation and characterization of biological samples and analytes. Among those methods, microdialysis gained its prominence due to its reliability in measuring various endogenous and exogenous therapeutic agents in various tissue systems. Virtually every soluble molecule in the interstitial fluid can be measured by microdialysis. Microdialysis facilitates inexpensive collection of accurate data with minimal invasive procedures. The present study describes the underlying principle of the technique, surgical method of probe insertion and sample collection from the target site. Further, we will describe the different components of the microdialysis instrument and its applications in various disciplines. Initially, microdialysis was designed to measure concentrations of neurotransmitters in rat brain. However, microdialysis has been adopted as a key research tool in several research areas to date. The first published application of microdialysis in humans was a study on interstitial glucose in 1987 and its use was initially confined to adipose tissue. However, numerous reports have since appeared on microdialysis in other human tissues such as brain, heart, lung, and solid tumors. The novelty of the present study lies in the use of microdialysis in rats which were treated with recurrent insulin injections for 14 days and adaptation of the technique for chronic collections of renal interstitial samples. In addition, this is the first report of the use of chronic microdialysis in a two week conscious animal protocol. Over the two week protocol, renal and interstitial samples can be collected and analyzed for changes in a number of different renal parameters.
\end{abstract}

Keywords: microdialysis, interstitial fluid, kidney, ATP, angiotensin II, renal hemodynamic

\section{Introduction}

Numerous studies have demonstrated the importance of changes in renal parameters to the control of blood pressure. Various renal parameters can be measured from blood such as creatinine in serum and blood urea nitrogen to evaluate alterations in renal function. However, to measure changes in renal agents for instance adenosine triphosphate (ATP) and angiotensin II (AngII), renal interstitial fluid must be sampled. Interstitial fluid or tissue fluid surrounds the cells and contains many components such as amino acids, sugars, fatty acids, hormones, neurotransmitters, and salts. Interstitial fluid is very similar to plasma. Thus, by analyzing the interstitial fluid, we can assess the tissue concentrations of various substances including drugs and peptides, whereas tissues are a major site for various biochemical and pharmacological events (Hansen et al., 1999). Thus, it would be clinically beneficial to obtain information from interstitial fluid rather than from blood. Many trails were performed to observe the chemical dynamics in the tissue, which includes several in vitro techniques (Ungerstedt, 1986) and various in vivo techniques (Chaurasia, 1999). In order to overcome the demerits of previously used push-pull techniques, further advancements were made on existing techniques, which resulted in the introduction of a new in vivo method namely, microdialysis, in which we can collect the tissue perfusate from the tissue of interest. This idea was first implemented by Delgado (1971) and then by Ungerstedt and Pycock (1974); where they used hollow fibers to infuse the perfusate continuously into the tissue and to collect it. Most current studies that utilize isolated tissues require one or more animals per time point, which provides 
general information about metabolism. However, prior to microdialysis many studies did not provide specific details about enzymes, intermediates or in vivo metabolic pathways. Therefore, previous traditional approaches, demonstrated far less correlation between in vitro and in vivo results (Hansen et al., 1999). These complications were overcome by microdialysis.

ATP, which is the source of internal energy, was demonstrated to have a paracrine role in the kidney (Nishiyama et al., 2004). Drury and Szent Gyorgyi (Drury et al., 1929) first observed the influence of extracellular purines on cardiovascular function. They reported that, by maintaining renal perfusion pressure, there was a marked reduction in renal blood flow (RBF) and urine flow by adenosine monophosphate (AMP) and adenosine. This resulted in increased attention on the role of adenosine in the control of renal hemodynamics and function. In order to affect renal blood flow the extracellular ATP would most likely have to alter afferent arteriole resistance (Nishiyama et al., 2004), therefore one could measure the ATP by collecting the renal interstitial fluid. Therefore, microdialysis has been used to measure renal ATP and AngII in acute animal models (Nishiyama et al., 2006). However, in a recently developed animal model of type 1 diabetes we were interested in evaluating the changes in renal ATP and AngII in a chronic setting. Thus, the previously utilized acute microdialysis technique has been modified to allow for chronic measurements over time.

Renin angiotensin system regulates the body's long term blood pressure and fluid balance by working together with the kidneys. When the blood volume is low, kidneys secrete renin. This renin converts angiotensinogen released by liver to angiotensin I which in turn is converted to angiotensin II by angiotensin converting enzyme present in lungs. This is the classically described renin angiotensin aldosterone system, where recent reports describe the existence of a complete angiotensin system in the kidney (Navar et al., 2011). Angiotensin II thus plays a critical role in maintaining blood pressure by its vasoconstrictive function. It stimulates aldosterone secretion from the adrenal cortex and release of $\mathrm{ADH}$ (anti-diuretic hormone) from the pituitary gland. These hormones help in increasing the blood volume by reabsorbing more sodium and water from urine. This ultimately results in increased blood pressure. If blood pressure exceeds the normal range then a negative feedback mechanism controls renin release which in turn inhibits angiotensin II conversion thus maintaining blood pressure. It was previously proposed that acute angiotensin II infusion promoted hypertension via induction in HO-I (Hemeoxygenase-I) by increasing CO levels. Inhibition of HO-I with ZnDPBG (zinc deuteroporphyrin 2,4-bis glycol) decreased blood pressure indicating a relationship between HO-I and blood pressure (Syed quadric et al., unpublished). Recurrent insulin doses resulted in hypertension. This was not controlled by negative feedback loop ultimately resulting in persistent hypertension .Thus the current study was performed to evaluate the hypothesis that this inhibition in the feedback mechanism of angiotensin II was due to renal ATP. To evaluate this hypothesis we analyzed ATP and angiotensin II levels in interstitial fluid using the developed chronic microdialysis technique (Figure 1).

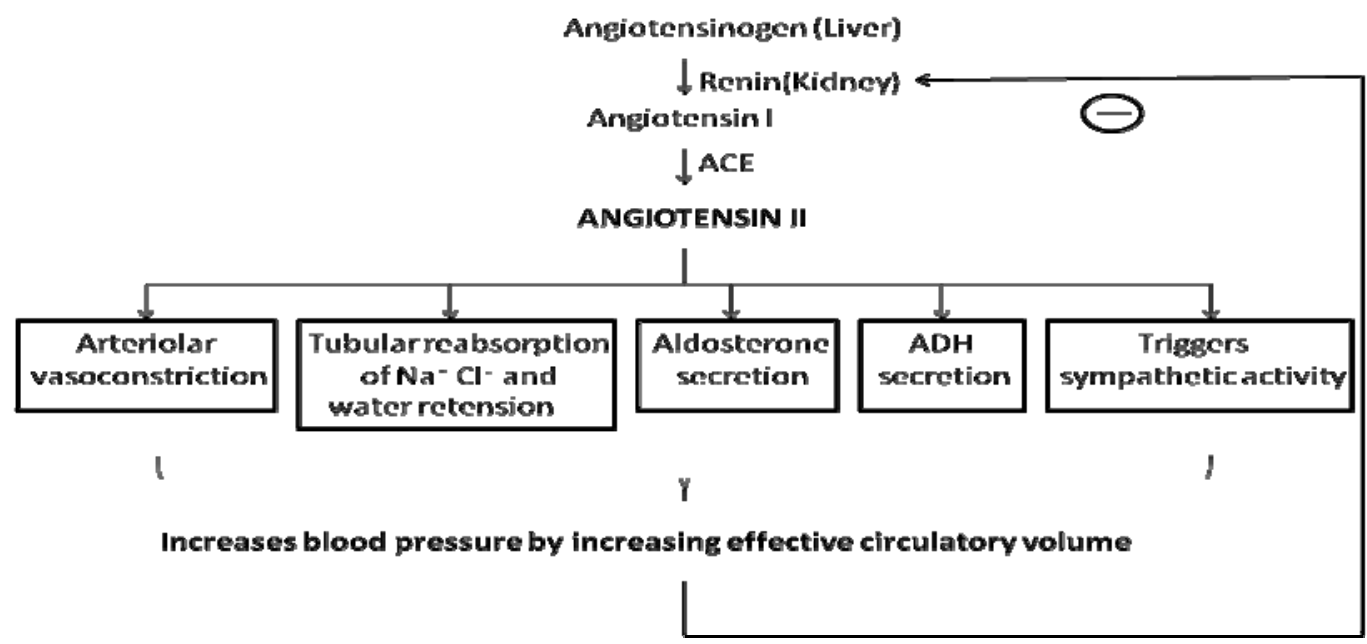

Figure 1. Role of Angiotensin II in maintaining blood pressure

Angiotensin II which is formed from Angiotensin I by the action of ACE (angiotensin coverting enzyme) maintains blood pressure in the body by various pathways as previously described. In case of high blood pressure it inhibits renin by a negative feedback mechanism. 


\section{Microdialysis Technique}

\subsection{Principle}

The basic idea of introducing a microdialysis probe is to imitate the blood vessel inside the tissue (Ungerstedt, 1986). The microdialysis technique is based on the concept of dialysis (Greek: to separate), where a semi-permeable membrane is introduced into the tissue of interest (Chaurasia, 1999). Thus, by introducing perfusate into the tubing, (Ungerstedt, 1986) there will be a bidirectional movement of molecules and ions between perfusate and the surrounding tissue, which depends on the solute concentration gradient (Jackson et al., 2000) (Chaurasia et al., 2007). This allows for the use of the probe for delivery, as well as, for sampling. Depending on the site of microdialysis, different types of probes are used which includes linear, loop, side-by-side and concentric probes (Chaurasia, 1999). In the present study, we used a linear probe, which contains a hollow fiber with a semi-permeable membrane in between. Thus, the tubing is inserted into the tissue of interest and positioned precisely at the site of interest, allowing the interstitial fluid to pass through it. The composition of the perfusion medium should closely match that of the extracellular fluids in the tissue of interest (Chaurasia, 1999). Solutes enter into the perfusate through the membrane due to the difference in concentration gradient, i.e. through simple diffusion (Figure 2). The semi-permeable membrane of the probe should be selected so that its pores are large enough to allow the movement of solute molecules of interest, yet small enough to resist proteins and other macromolecules (Chaurasia, 1999). In this light the microdialysis probe acts as a primary filter to sample the interstitial fluid for the substance of interest. To serve this purpose, different types of semi-permeable membranes are employed, which include polycarbonate-ether, regenerated cellulose, polyacrylonitrile (PAN) (Hsiao et al., 1990) and polyether sulfone (PES) (Elmquist \& Sawchuk, 1997). Since this technique has no actual loss or gain of body fluids; except for the exchange of ions, it is said to be a "volume neutral" technique (Chaurasia et al., 2007). Microdialysis probes are calibrated (Lonnroth et al., 1987) to establish the equilibrium between perfusate and interstitium. This technique can also be applied for other purposes for example, evaluating the enzymatic conversion of a particular precursor by introducing it into the tissue and collecting the perfusate to analyze it.

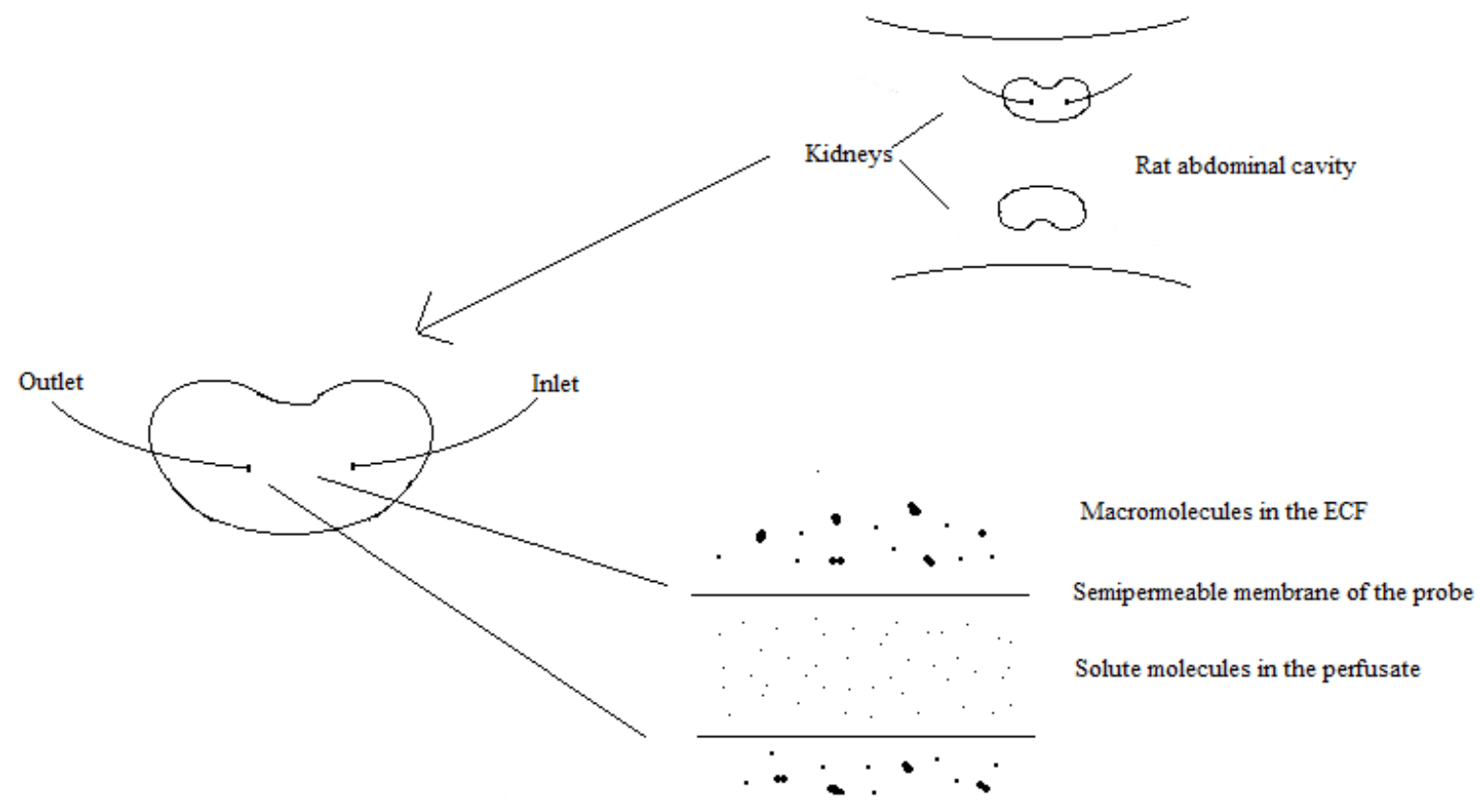

Figure 2. Probe positioning in the kidney

This figure represents the placement of the probe inside the kidney. The probe present in the kidney allows bidirectional movement of selective molecules and ions.

\subsection{Merits}

-Samples can be collected from intact tissues of freely moving animals, thereby mimicking the normal condition. 
-The same animal from which the samples are collected acts as its own control which reduces the inter subject variability (Meeusen et al., 2001).

-The semi-permeable nature of the probe prevents the intervention of bigger molecules like proteins in the perfusate, which helps in collecting a clear solution for analysis.

-No loss in body fluids aids both acute and chronic studies.

-Tissue irritation will be minimal due to perfusate, since it does not come into direct contact with the tissue.

-Probes can be inserted in any part of the organ or the tissue.

-Multiple sites can be used for analysis (though focal information on tissue PK is limited) (Chaurasia et al., 2007).

-Probe insertion is similar to that of intravenous catheter but, with minimal pain.

\subsection{Demerits}

-Drug associated problems may be encountered.

-Analysis should be performed very efficiently due to the small amount of sample collected.

-Surgical skills are required for correct placement of the probe.

-Recovery time is variable.

2.4 Applications

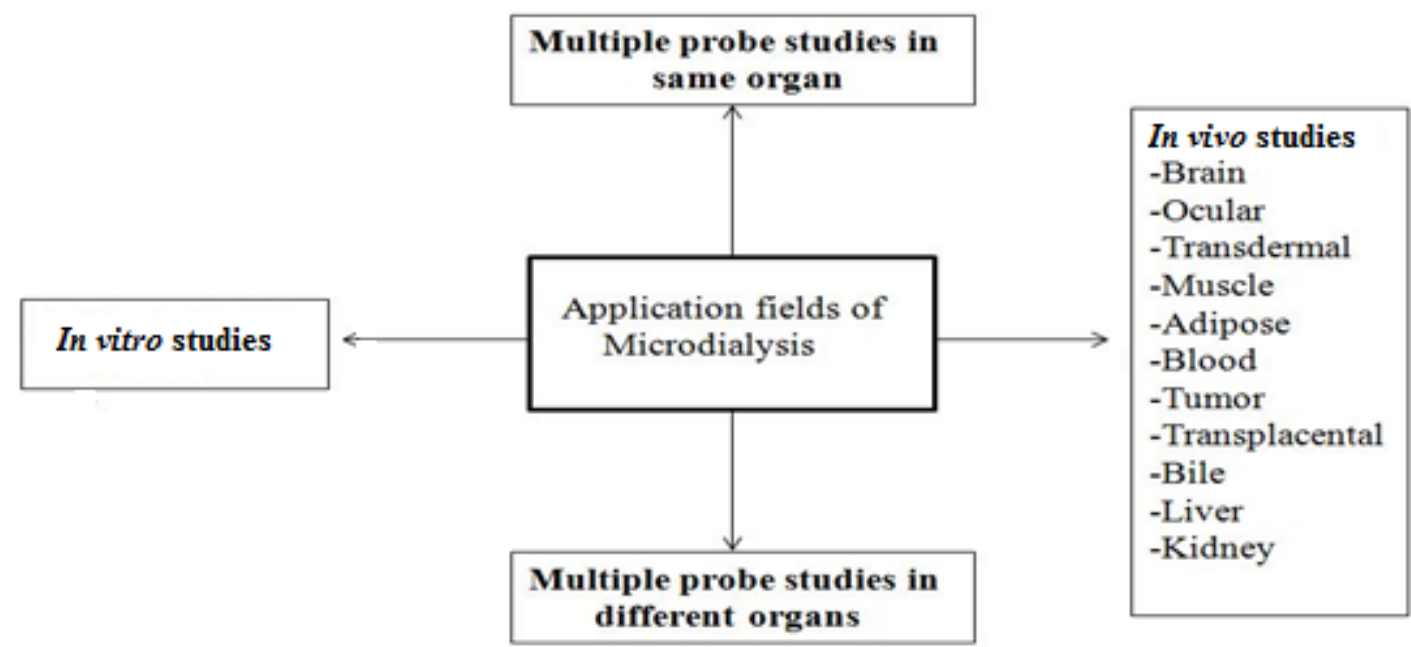

Figure 3. Applications of microdialysis in various disciplines

Microdialysis technique facilitates various in vitro and in vivo studies along with research involving the same and different organ systems.

\subsection{Instrumentation and Setup}

The microdialysis setup allows one to perform unattended experiments in rats. Samples can be colllected either manually or automatically. Injections can also be included along with microdialysis. The instrument (obtained from CMA, a Harvard apparatus company) mainly consists of the following components (Figure 4):

1) Syringe pump

2) Microfiltration collector

3) Micro $T$

4) Assembly for freely moving animals (bowl, swivel assembly and balance arm) 


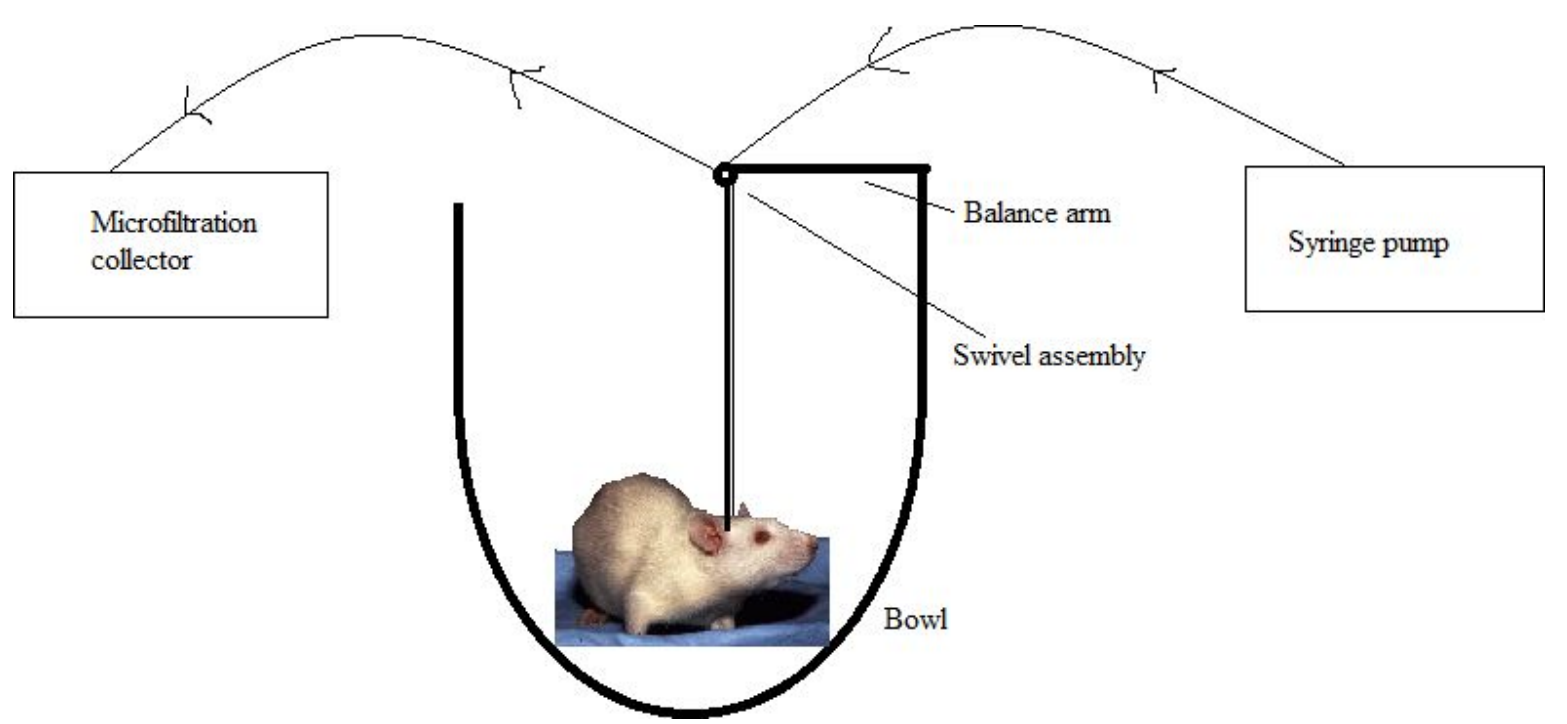

Figure 4. Instrumental setup for chronic awake animal studies

This gives the pictorial representation of the instrumental setup for perfusate infusion through syringe pump and dialysate collection through microfiltration collector. The swivel assembly allows for free movement of the animal.

\subsubsection{Syringe Pump}

The syringe pump can control dual syringes independently with low, pulse-free flow, which is maintained between $0.1 \mu \mathrm{l} / \mathrm{min}$ and $20 \mu \mathrm{l} / \mathrm{min}$. The flow direction can be easily reversed individually for the syringes. Thus, allowing for sampling of the interstitial fluid and/or drug delivery.

\subsubsection{Micro Fraction Collector}

The micro fraction collector is a compact single or dual fraction collector which aides in micro sampling. It can collect volumes of 1 to $50 \mu \mathrm{l}$ from 1 or 2 microdialysis probes. The samples are collected from the outlet tubing of the probe, which acts as collection cannula. The samples are collected into vials which are refrigerated pending analysis. The micro fraction collector is quiet, small and promotes no animal disturbances; therefore it can be placed near the animals.

\subsubsection{System for Free Movement of the Animal}

This technique can be used in conscious, freely moving animals. It can be used for both chronic and acute studies. The unrestricted movement of the animals enables performance of experiments for longer periods of time without disturbing the animals. The balance arm attached to the cage or CMA instrument table, has a dual channel swivel pivoted to the edge of the bowl, in order to follow the animal's movement closely. Tension on the microdialysis probe is prevented by attaching the swivel, which is pulled by a steel wire to a collar on the animal. The animal is placed in a round bottom bowl; with free access to food and water.

\subsubsection{Micro T}

Micro $\mathrm{T}$ is a 3 way connector which is connected to a syringe pump and a microdialysis probe. This minimizes the potential of introducing air bubbles into the perfusion line during syringe changes.

\section{Experimental Procedure}

\subsection{Animal Model}

A total of 10 adult male Sprague-Dawley rats (200-250 g) were housed in a colony maintained at room temperature. They were provided free access to food and water throughout the experiment. All animal experiments were approved by the University of Louisiana at Monroe Institutional Animal Care and Use Committee (IUCAC).

\subsection{Implanting the Probe}

Animals were anesthetized using an isoflourane anesthesia setup (Ez-anesthesia system) and animals were anesthetized throughout the surgical procedure. After a reflex test was performed to evaluate proper animal 
anesthesia level, rats were positioned for surgery. Rough markings were made on the nape of the neck for inlet and outlet ports of the probe and over the area of the kidney. A midsagittal incision was made to excise the kidney. A small hollow tubular structure (open at both ends which helps us to insert the probe tubing) was inserted through this incision and slowly moved underneath the skin to the nape of the neck. A small incision was made to allow the tube to exit. The microdialysis probe of $10 \mathrm{~mm}$ membrane length, with membrane outer diameter of $0.24 \mathrm{~mm}$ and molecular weight cutoff of 6000 Daltons, was then inserted into the hollow tube. Once it was out from the other end, it was inserted into the kidney with the help of a $221 / 2 \mathrm{G}$ needle. Great care was taken to ensure that the probe was inserted into the kidney in one attempt in order to prevent internal rupture or bleeding of the organ. This allows for stability of the animal during the 2 week study. After probe insertion, it was passed back through the same hollow tube and exited at the nape of the neck. Thus the inlet and outlet ports are through the same opening made at the nape of the neck (minimizing injury and stitches). Once the tubing was removed, visual inspection was performed to ensure proper placement of the dialysis window inside of the kidney. If required, adjustment of the dialysis window was accomplished through proximal or distal movement of the probe. Once the probe was fixed, the incisions (both at the neck and on the side) were sutured. Insertion of the probe with minimal invasiveness was a technique adopted from (Siragy et al., 1997). Siragy and Carey inserted probes into 2 kidneys and samples were collected for 4 days. Their technique was modified as detailed below to allow collection of samples for 14 days without disturbing the animal. Hydrogen peroxide was applied at the suture site to prevent any further infection. The animal was then placed in the cage with the microdialysis setup. The inlet port was connected to the syringe pump (CMA 420) and the outlet tubing was fixed to the micro fraction collector (CMA 142). Heparinized saline was flushed through the tubing to make sure that the tubing is not blocked. The flow rate was maintained at $1 \mathrm{ml} / \mathrm{hr}$. All animals recovered and regained their weight after 1-2 days post surgery.

\subsection{Symptoms of Ineffective Surgery}

-Internal bleeding can be observed through the presence of blood in the urine.

-Bleeding around the sutured areas.

-Signs of visual animal stress, such as lack of movement in the cage or through vocal sounds of distress.

\subsection{Insulin Treatment}

The animal is given 2-3 days to recover. After the recovery period, the animal is treated with a daily $7 \mathrm{U} / \mathrm{Kg}$ dose of insulin (subcutaneous). Samples are collected 1 hour post insulin injection for a period of 2 hours.

\subsection{Collection of Samples}

Samples were collected at 3 intervals of 2 hours each day. Therefore, three $2 \mathrm{ml}$ samples were collected and stored at $-80^{\circ} \mathrm{C}$ until analyzed. Flushing was required before collecting the samples, to prevent coagulation around the tubing.

\subsection{Analysis of the Samples}

Collected samples were analyzed for ATP and angiotensin II. ATP levels were determined using luciferin-luciferase assay (Nishiyama et al., 2006). Angiotensin II levels were determined via commercially available ELISA kits (Sigma Aldrich).

Table 1. ATP Bioluminescent Assay

\begin{tabular}{cc}
\hline Time & Concentration of ATP in samples $(\mathrm{ng} / \mu \mathrm{l})$ \\
\hline DAY 5 & 22.95 \\
DAY 6 & 26.57 \\
\hline
\end{tabular}

Table 2. Angiotensin II ELISA

\begin{tabular}{cc}
\hline Time & Concentration of Ang II (pg/ml) \\
\hline Day 5 & 2.54 \\
Day 6 & 0.96 \\
\hline
\end{tabular}




\subsection{Results}

In a small subset of animals, renal microdialysis samples were analyzed to determine the concentration of ATP and angiotensin II (Figure 5).

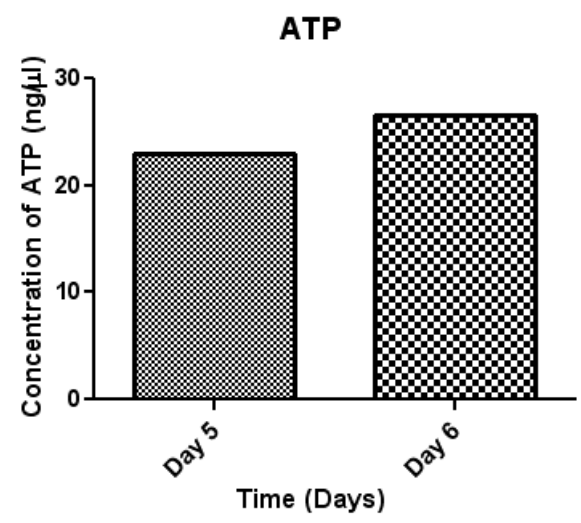

Figure 5. Analysis of ATP concentration

ATP concentrations were analyzed in the interstitial fluid collected from the animals during day 5 and day 6 by using luciferin-luciferase assay.

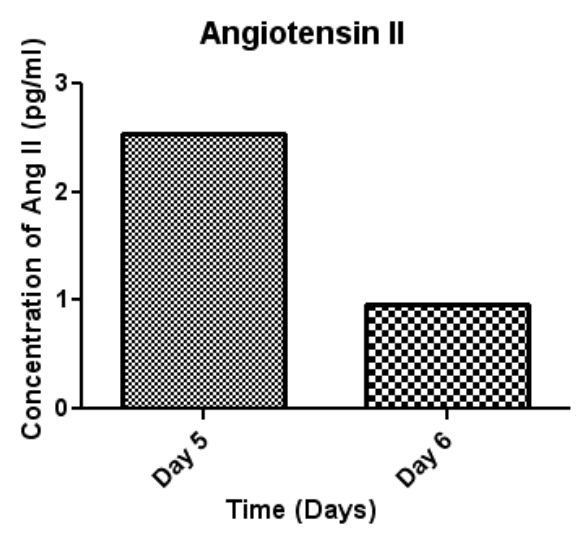

Figure 6. Analysis of Ang II concentration

Ang II concentrations were analyzed in the interstitial fluid collected from animals during day 5 and day 6 by using ELISA.

\section{Discussion}

Microdialysis technique, which was initially only used for brain research has been adopted in various disciplines and tissues including human studies. Microdialysis permits collection of clear samples, free from large molecules (proteins) without any special cleanup procedure. During drug administration, microdialysis allows for continuous monitoring of various processes for example absorption, distribution, and metabolism, which are present at various tissue sites such as in the liver, kidney, brain, and muscle. In addition, during drug administration, microdialysis permits concurrent sampling of various fluids for instance in blood, bile, and brain extracellular fluids. This technique facilitates the use of a minimum number of animals; in that one can get continuous samples for several days without any change (loss or gain) in body fluids of the animal. Samples can be simultaneously collected from multiple sites of the same animal (Muller, 2002). This sampling technique avoids the volume limitations associated with small animal pharmacokinetics (Nishiyama et al., 2004). Given the fact that samples can be collected before, during and after treatments each animal can serve as its own control. Therefore, microdialysis can assist in conducting cross over experiments with a single animal. In this technique, there is no further risk of enzymatic degradation, as proteins and other macromolecules are entirely separated, 
once perfusate is collected from the tubing. The outstanding feature of microdialysis is that it allows for direct comparison of in vitro and in vivo experiments. By using an appropriate size membrane and a sufficiently low perfusion speed, it is possible to reach $100 \%$ recovery. Hence one can accurately approximate the extracellular concentration of various compounds.

Microdialysis is a prominent tool in drug development, where selection of an appropriate compound for further development and optimization of dosing regimens is invaluable. In this regard, FDA centre of devices and radiological health has approved 2 devices using the principle of microdialysis; a cerebral tissue monitoring system to monitor biochemical markers of ischemia in the brain (Chaurasia et al., 2007), and another device to measure glucose through the skin (Klonoff, 2005). Clinical microdialysis has been shown to be a safe, reproducible, ethically acceptable and relatively inexpensive technique for studying tissue biochemistry and drug distribution in humans (Ungerstedt, 1974; Lonnroth et al., 1995; Muller, 2000; Muller, 2002). To date microdialysis is becoming an established clinical technology that can be applied to most organs in appropriate clinical situations (Chaurasia et al., 2007).

The uniqueness of this experiment lies in the less invasive method used to insert the probe into the animal's body. The probe is inserted by making a small incision. In the past, the probes were inserted through a large abdominal opening in the animal which is considered to be a highly invasive technique. Previous insertion methods increased the recovery time for the animal under study and also rendered the animal unstable in a relatively short time frame.

The rational for the current study is that it permits study of the animal for 14 days without hampering the animal's activities. The less intrusive technique also allows the animal to recover quickly, without any complications being developed over the 14 days in which the experiment is conducted. Thus we can collect the samples from the probe whenever required without disturbing the animal and its movements.

There was a significant increase in renal AngII and ATP during the recurrent insulin induced hypoglycemic protocol. Hence by using the previously described microdialysis technique elevated AngII levels and chronic changes in ATP can be monitored.

\section{References}

Bolinder, J. et al. (1993). Long-term continuous glucose monitoring with microdialysis in ambulatory $\begin{array}{llll}\text { insulin-dependent diabetic } & \text { patients. }\end{array}$ http://dx.doi.org/10.1016/0140-6736(93)92063-y

Chaurasia, C. S. (1999). In vivo microdialysis sampling: theory and applications. Biomed Chromatogr, 13(5), 317-332.

Chaurasia, C. S. et al. (2007). AAPS-FDA workshop white paper: microdialysis principles, application and regulatory perspectives. Pharm Res., 24(5), 1014-1025. http://dx.doi.org/10.1007/S11095-006-9206-Z

Delgado, J. M. et al. (1971). Brain excitability and behavioral reactivity in monkeys under meprobamate. Arch Int Pharmacodyn Ther., 194(1), 5-17.

Drury, A. N., \& Szent-Gyorgyi, A. (1929). The physiological activity of adenine compounds with especial reference to their action upon the mammalian heart. J. Physiol., 68(3), 213-237.

During, M. J., \& Spencer, D. D. (1993). Extracellular hippocampal glutamate and spontaneous seizure in the conscious human brain. Lancet, 341(8861), 1607-1610. http://dx.doi.org/10.1016/0140-6736(93)90754-5

Elmquist, W. F., \& Sawchuk, R. J. (1997). Application of microdialysis in pharmacokinetic studies. Pharm Res., 14(3), 267-288. http://dx.doi.org/10.1023/A:1012081501464

Hansen, D. K., et al. (1999). Pharmacokinetic and metabolism studies using microdialysis sampling. J. Pharm. Sci, 88(1), 14-27. http://dx.doi.org/10.1021/JS9801485

Herkner, H. et al. (2002). Closed-chest microdialysis to measure antibiotic penetration into human lung tissue. Am. J. Respir. Crit. Care. Med., 165(2), 273-276.

Hsiao, J. K. et al. (1990). Effects of different semipermeable membranes on in vitro and in vivo performance of

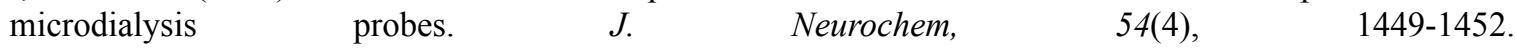
http://dx.doi.org/10.1111/J.1471-4159.1990.TB01982.X

Jackson, K. et al. (2000). Cardiac microdialysis a powerful tool. Cardiovasc Res., 46(3), 367-369. http://dx.doi.org/10.1061/S0008-6363(00)00085-7.

Kennergren, C. et al. (1999). Monitoring of extracellular aspartate aminotransferase and troponin T by 
microdialysis during and after cardioplegic heart arrest. Cardiology, 92(3), 162-170. http://dx.doi.org/10.1159/000006966

Klonoff, D. C. (2005). Continuous glucose monitoring: roadmap for 21 st century diabetes therapy. Diabetes Care, 28(5), 1231-1239. http://dx.doi.org/10.2337/diacare.28.5.1231

Lonnroth, P., \& Strindberg, L. (1995). Validation of the 'internal reference technique' for calibrating microdialysis catheters in situ. Acta Physiol Scand, 153(4), 375-380.

Lonnroth, P. et al. (1987). A microdialysis method allowing characterization of intercellular water space in humans. Am. J. Physiol., 253(2 Pt 1), E228-231.

Meeusen, R. et al. (2001). Brain microdialysis in exercise research. Sports Med, 31(14), 965-983.

Muller, M. (2000). Microdialysis in clinical drug delivery studies. Adv Drug Deliv Rev., 45, 255-269

Muller, M. (2002). Science, medicine, and the future: Microdialysis. BMJ, 324(7337), 588-591.

Muller, M. et al. (1997). 5-fluorouracil kinetics in the interstitial tumor space: clinical response in breast cancer patients. Cancer Res., 57(13), 2598-2601.

Navar, L. G. et al. (2011) Intrarenal angiotensin II and its contribution to the genesis of chronic hypertension. Curr Opin Pharmacol, 11(2), 180-186.

Nishiyama, A. et al. (2004). Role of interstitial ATP and adenosine in the regulation of renal hemodynamics and microvascular function. Hypertens Res., 27(11), 791-804. http://dx.doi.org/10.1152/ajheart.00242.2005

Nishiyama, A. et al. (2006). Renal interstitial fluid ATP responses to arterial pressure and tubuloglomerular feedback activation during calcium channel blockade. Am J. Physiol Heart Circ Physiol, 290(2), H772-777.

Siragy, H. M., \& Carey, R. M. (1997). The subtype 2 angiotensin receptor regulates renal prostaglandin F2 alpha formation in conscious rats. Am J. Physiol, 273(3 Pt 2), R1103-1107.

Ungerstedt, U. (1986). Microdialysis- A new bioanalytical sampling technique. Curr Separations, 7, 43-46

Ungerstedt, U. (1991). Microdialysis--principles and applications for studies in animals and man. J. Intern Med, $230(4), 365-373$.

Ungerstedt, U., \& Pycock, C. (1974). Functional correlates of dopamine neurotransmission. Bull Schweiz Akad Med Wiss, 30(1-3), 44-55. 